Editorial

\title{
Acknowledgement to Reviewers of Inorganics in 2018
}

\section{Inorganics Editorial Office}

MDPI, St. Alban-Anlage 66, 4052 Basel, Switzerland

Published: 9 January 2019

Rigorous peer-review is the corner-stone of high-quality academic publishing. The editorial team greatly appreciates the reviewers who contributed their knowledge and expertise to the journal's editorial process over the past 12 months. In 2018, a total of 125 papers were published in the journal, with a median time to first decision of 15 days and a median time to publication of 36 days. The editors would like to express their sincere gratitude to the following reviewers for their cooperation and dedication in 2018:

Adonin, Sergey A.

Akitsu, Takashiro

Aldrich-Wright, Janice

Aldridge, Simon

Alonso, Francisco

Amouri, Hani

Aquino, Adelia J. A.

Argitis, Panagiotis

Atuchin, Victor V.

Ballato, John

Bandala, Erick R.

Bardin, Vadim V.

Barišić, Lidija

Barolo, Claudia

Bauer, Eike

Bella, Federico

Bendix, Jesper

Bermejo, María Dolores

Best, Stephen

Beyeh, Ngong Kodiah

Bizzarri, Claudia

Bokach, Nadezhda

Bominaar, Emile L.

Borbas, K. Eszter

Bossmann, Stefan

Bruce, Alice

Brusso, Jaclyn L.

Brutti, Sergio

Cámpora, Juan
Capacchione, Carmine

Carniato, Fabio

Cerrada, Elena

Champagne, Benoît

Charmet, Andrea Pietropolli

Chavez, Ferman

Chen, Lung-Chien

Chiang, Ming-Hsi

Chippindale, Ann

Choi, Kwang-Yong

Chorazy, Szymon

$\mathrm{Chu}$, Jiaxiang

Ciancaleoni, Gianluca

Colombo, Alessia

Condorelli, Daniele Filippo

Constantino, Ferdinando

Corey, Joyce Y.

Cosquer, Goulven

Costa, Paulo Jorge

Costisor, Otilia

Crans, Debbie

Csámpai, Antal

De Munno, Giovanni

Delaude, Lionel

Delcamp, Jared

Della Ca', Nicola

Demadis, Konstantinos D.

Di Bella, Santo

Dixit, Mudit 
Dixon, David

Dos Santos-García, Antonio J.

Dragonetti, Claudia

Du, Yanhai

Dutton, Jason L.

Duttwyler, Simon

Dvořák, Zdeněk

Elliott, Paul

Errandonea, Daniel

Faller, Peter

Fässler, Thomas F.

Fedorov, Pavel

Fernandes, Alexandra R.

Fielden, John

Filippou, Alexander C.

Filippov, Oleg A.

Florindo, Pedro R.

Fornasiero, Paolo

Freitag, Marina

Frontera, Antonio

Fujita, Ken-ichi

Gambino, Dinorah

Garcia, Yann

García, Juan

García, Begoña

Garroni, Sebastiano

GASSIN, Pierre-Marie

Genty, Eric

Gerasimchuk, Nikolay

Gesing, Thorsten M.

Giannici, Francesco

Glazer, Edith C.

Gómez-Ruiz, Santiago

Gossage, Robert A.

Grin, Yuri

Gschwind, Fabienne

Guazzelli, Lorenzo

Gündoğ, Yücesan

Hadlington, Terrance

Hambley, Trevor

Hanif, Muhammad

Hannedouche, Jerome

Hashizume, Mineo
Hayashi, Yoshihito

Herres-Pawlis, Sonja

Hetmańczyk, Łukasz

Hevia, Eva

Higham, Lee J.

Hirva, Pipsa

Holder, Alvin A.

Hopkins, Todd A.

Hörner, Gerald

Hsu, Sodio C. N.

Inoue, Shigeyoshi

Ishida, Takayuki

Ishikawa, Ryuta

Iwamura, Munetaka

Izarova, Natalya V.

Izod, Keith

Jensen, Torben

Jing, Huanwang

Johnson, Samuel A.

Jubera, Véronique

Jutzi, Peter

Kaczmarek, Anna M.

Kageyama, Hiroshi

Kalayda, Ganna

Kaluderovic, Goran

Kameo, Hajime

Katoh, Keiichi

Khandelwal, Manish

Kirillov, Alexander

Klein, Axel

Koduru, Janardhan Reddy

Kowalski, Konrad

Kraus, Florian

Krukowski, Stanislaw

Kukli, Kaupo

Kurouski, Dmitry

Kuwata, Shigeki

Laguna, Antonio

Lamac, Martin

Langer, Robert

Lao, Ka Un

Le Guennic, Boris

Lee, Sonny 
Lemercier, Gilles

Leonidovich Kuzemsky, Alexander

Lewkowski, Jarosław A.

Li, Guanchen

Li, Mingqiang

Liddle, Steve

Lin, Po-Heng

Linert, Wolfgang

Liu, Shiuh-Tzung

Liu, Shi-Xia

Liu, Tianbo

Longo, Pasquale

Lucenti, Elena

Łukasik, Rafał

Luo, Yi

Luzyanin, Konstantin

Macak, Jan M.

Madalan, Augustin M.

Magni, Mirko

Maireles Torres, Pedro

Malandrino, Graziella

Manoury, Eric

Marchetti, Fabio

Marnellos, George

Marschner, Christoph

Martínez, Cristina

Martins, Luísa Margarida

Matesanz, Ana I.

Matoga, Dariusz

McFarland, Sherri

McMorran, David

McNamara, Bruce

Medici, Serenella

Meier-Menches, Samuel M.

Merlino, Antonello

Merten, Christian

Mewis, Ryan E.

Michel, Sarah L. J.

Minakshi, Manickam

Miró, Eduardo E.

Mitoraj, Mariusz

Moineau-Chane-Ching, Kathleen I.

Mosquera, Marta
Mota, Antonio J.

Müller, Danny

Murray, Keith

Musiol, Robert

Navalon, Sergio

Nazarov, Alexey

Nemec, Ivan

Nemykin, Victor

Nesterov, Dmytro

Neves, Maria

Newton, Graham N.

Niewa, Rainer

Nippe, Michael

Nisnevitch, Marina

Ohkubo, Isao

Olmos, Dania

Palinko, Istvan

Papurello, Davide

Parac-Vogt, Tatjana N.

Paraskevopoulou, Patrina

Park, Sung Ha

Pawluć, Piotr

Pérez-Moreno, Javier

Perlepes, Spyros P.

Perrier, Aurélie

Perry, Nicola

Pichon, Céline

Pietschnig, Rudolf

Pinkowicz, Dawid

Pitsikalis, Marinos

Poater, Albert

Poddel'sky, Andrey

Pointillart, Fabrice

Prashar, Sanjiv

Puchkova, Ludmila V.

Reber, Christian

Redshaw, Carl

Ribbe, Markus W.

Rinehart, Jeffrey

Roberto, Dominique

Roll, Mark

Romero-Canelón, Isolda

Roşca, Sorin-Claudiu 
Ruiz, Miguel A.

Ryde, Ulf

Sadykov, Vladislav

Saielli, Giacomo

Sakon, Takuo

Saleev, Vladimir

Samia, Benmansour

Santoni, Marie-Pierre

Sarazin, Yann

Sasamori, Takahiro

Scheiner, Steve

Schmedake, Thomas A.

Schneider, Raphaël

Schnepf, Andreas

Schoen, Christian

Seib, Philipp

Serwicka, Ewa M.

Setlur, Anant A.

Sheehan, Stafford W.

Shenderovich, Ilya G.

Siegbahn, Per E. M.

Snegur, Lubov

Sorace, Lorenzo

Speghini, Adolfo

Spingler, Bernhard

Stasch, Andreas

Stasiuk, Graeme

Stoikov, Ivan Ivanovich

Suaud, Nicolas

Sun, Luyi

Suntharalingam, Kogularamanan

Svensson, Gunnar

Szeghalmi, Adriana

Szilágyi, Istvan

Szilvási, Tibor

Takao, Toshiro

Takeshita, Satoru

Tenne, Reshef
Toroker, Maytal Caspary

Tóth, Éva

Tran, Fabien

Trindle, Carl

Trujillo-Rodríguez, María José

Trusso Sfrazzetto, Giuseppe

Tsipis, Athanassios C.

Tsipis, Ekaterina V.

Tsukerblat, Boris

Ueda, Tadaharu

Urbani, Maxence

Valyaev, Dmitry A.

Van Der Vlugt, Jarl Ivar

Vaňura, Petr

Varadwaj, Pradeep R.

Vicic, David

Villanneau, Richard

Vostrikova, Kira E.

Wada, Tohru

Wallis, John

Walsby, Charles

Wang, Wei

Wark, Michael

Watanabe, Motonori

Waterman, Rory

Weinberger, Peter

Westcott, Stephen A.

Wezenberg, Sander J.

Wilson, Justin J.

Wypych, Fernando

$\mathrm{Xu}$, Jian

Yamamoto, Yasunori

Yamane, Hisanori

Yeh, Chen-Yu

Yoshimura, Kazuyoshi

Zanotti, Valerio

Zierkiewicz, Wiktor

(C) 2019 by the authors. Licensee MDPI, Basel, Switzerland. This article is an open access article distributed under the terms and conditions of the Creative Commons Attribution (CC BY) license (http://creativecommons.org/licenses/by/4.0). 